Bio-grafía: Escritos sobre la Biologia y su Enseñanza Vol. 5 No 9. Monográfico de Evolución. ISSN 2027-1034. Jutio a Diciembre del 2012 P. p. 4-18.

\title{
LA EXTENSIÓN DE LA SÍNTESIS EVOLUTIVA Y LOS ALCANCES SOBRE LA ENSEÑANZA DE LA TEORÍA DE LA EVOLUCIÓN
}

\author{
THE SPREAD OF EVOLUTIONARY SYNTHESIS AND THE REACHES ABOUT \\ EVOLUTION THEORY TEACHING
}

\section{Por: Guillermo Folguera ${ }^{1}$ y Leonardo Galli ${ }^{2}$}

\author{
Recibido: 01-10-2012 \\ Aceptado: 11-12-2012
}

\section{Resumen}

Entre las décadas de 1930 y 1940 se generó el consenso que dio el origen a la denominada síntesis evolutiva. Este consenso presentaba un conjunto teórico de gran riqueza a la vez que se proponía como un programa de investigación para el estudio de la evolución de la vida. Sin embargo, en las últimas décadas algunos aspectos de este conjunto teórico fueron cuestionados, a la vez que otras áreas de investigación fueron incorporadas, dando lugar a una serie de complejos debates de orden diverso. Por otro lado, en relación con el ámbito educativo, existe un amplio acuerdo sobre la necesidad de que la enseñanza secundaria obligatoria garantice un sólido aprendizaje de los modelos y conceptos fundamentales de la biología evolutiva, al tiempo que se reconoce que los resultados, en términos de aprendizaje, son muy deficientes. En dicho contexto surge la cuestión de qué modelos de la biología evolutiva deberían servir como referencia para la enseñanza obligatoria. En este trabajo analizamos diferentes argumentaciones en relación con la inclusión o no de las últimas modificaciones teóricas en la enseñanza de la teoría de la evolución en algunos ámbitos educativos. Con este fin, en una primera instancia analizamos algunas de las principales modificaciones y propuestas teóricas que han tenido lugar en relación con la teoría de la evolución de las últimas décadas. Posteriormente, indagamos algunas de las argumentaciones sobre su posible inclusión en el ámbito educativo. Por último presentamos algunos apuntes finales que hacen más las veces de apertura que de una búsqueda de conclusiones.

Palabras clave: evolución biológica, síntesis evolutiva, enseñanza de la biología evolutiva.

\section{Abstract}

Between the decades of 1930 and 1940, the agreement, which originated the so called evolutionary synthesis, was generated. This agreement had a very rich theoretical body, and at the same time, it was proposed as a research program for the study of the biological evolution. However, during the last four decades, some aspects of this theoretical body were questioned at the same time that other areas of research were incorporated, which gave rise to long, complicated debates of different nature. On the

\footnotetext{
${ }^{1}$ Grupo de Filosofía de las Ciencias, Facultad de Filosofía y Letras / Facultad de Ciencias Exactas y Naturales, Universidad de Buenos Aires. Concejo Nacional de Investigaciones Científicas y Técnicas.

${ }^{2}$ Grupo de Didáctica de la Biología, Instituto de Investigaciones CEFIEC, Facultad de Ciencias Exactas y Naturales, Universidad de Buenos Aires.
} 
other hand, regarding to the educational field, there is a great agreement about the necessity that the obligatory secondary teaching should guarantee a solid learning of the models and principal concepts of evolutionary biology, at the same time that it is recognized that the results, in terms of learning, are very poor. In this context, the question of which models of the evolutionary biology should work as a reference for the obligatory teaching emerges. In this work, we analyze different argumentations related to the inclusion or not of the latest theoretical changes in the evolutionary theory teaching, in the educational fields. With this purpose, first of all, we analyzed some of the principal changes and theoretical proposals which have taken place regarding to the evolutionary theory in the last decades. Subsequently, we have inquired some of the argumentations, about their possible inclusion in the educational field. Finally, we have made some final notes which mean more an opening than a search of conclusions.

Key words: biological evolution, evolutionary synthesis, evolutionary biology teaching.

\section{Introducción}

La expresión "teoría sintética de la evolución" deriva del libro publicado en 1942 por Julian Huxley, titulado Evolución, la síntesis moderna (Gould, 2004), y se refiere a un consenso de la comunidad científica alcanzado en las décadas de 1930 y 1940 respecto a la integración entre la teoría de Charles Darwin y las teorías de la genética (Futuyma, 2009; Mayr, 1992). Este consenso presentaba un conjunto teórico de gran riqueza, a la vez que se proponía como un programa de investigación para el estudio de la evolución de la vida. Sin embargo, en las últimas décadas algunos aspectos de este conjunto teórico fueron cuestionados, a la vez que otras áreas de investigación fueron incorporadas, tales como la macroevolución y la biología evolutiva del desarrollo (evo-devo). Evidentemente, estos escenarios han dado lugar a una serie de complejos debates de orden diverso y aún no están suficientemente claros los alcances de las modificaciones teóricas involucradas.

En relación con el ámbito educativo, existe un amplio acuerdo, tanto entre el profesorado como entre los investigadores en didáctica de la biología, sobre la necesidad de que la enseñanza secundaria obligatoria garantice un sólido aprendizaje de los modelos y conceptos fundamentales de la biología evolutiva (NAS 2004). Este consenso se debe al reconocimiento de que la teoría de la evolución ocupa un lugar central en la Biología, integrando todas las demás áreas de esta disciplina (Futuyma, 2009), y de que se trata de una teoría con profundas implicancias para muchas otras áreas disciplinares tales como la epistemología, la ética y la sociología (Ruse, 1994; Stamos, 2009). A pesar de esto, son numerosas las evidencias de que el resultado de la enseñanza en relación con estos contenidos está muy lejos de lo deseable (Alters y Nelson, 2002). Los factores que dificultan la enseñanza y el aprendizaje de la biología evolutiva son numerosos e incluyen la falta de conocimientos previos, la influencia de creencias religiosas, la inadecuación de los materiales didácticos, la inadecuada formación del profesorado y la existencia y persistencia de concepciones alternativas incompatibles con los modelos científicos (Smith 2010a, 2010b).

Una parte significativa de la bibliografía especializada señala que los problemas residen en los estudiantes o en los docentes (por una supuesta falta de conocimientos, por preconceptos) al tiempo que se asume que los modelos a enseñar son "a-problemáticos". Esto es, se asume que estos modelos están claramente formulados, sin ambigüedades ni contradicciones, sin zonas oscuras que requieran cierta elucidación. Por el contrario, 
consideramos que es necesario un análisis epistemológico de los modelos a enseñar a fin de detectar posibles "nudos de dificultad". En un sentido más elemental aún, es necesario preguntarse qué modelos conforman la teoría a enseñar para luego poder reflexionar sobre cuáles merecen ser incorporados en la educación general. Esta reflexión se hace particularmente necesaria en el caso de la biología evolutiva debido al estado de ampliación y revisión que está sufriendo la síntesis evolutiva $(\mathrm{SE})^{3}$, tomada habitualmente como referente para la enseñanza obligatoria. Así, en la primera parte de este artículo reseñamos dos conjuntos de cuestionamientos a los fundamentos teóricos de la SE para luego analizar algunas de las implicancias didácticas de estos debates, reconociendo elementos que podrían incidir en su inclusión o no en los ámbitos de la enseñanza de la teoría de la evolución. Por último, realizaremos algunos apuntes finales que esperamos que hagan las veces más de apertura que de una búsqueda de conclusiones.

\section{Los fundamentos de la síntesis evolutiva}

En primera instancia, recordemos algunos de los aspectos generales de la SE. En términos generales, la SE fue generada por la integración de dos conjuntos teóricos. La primera corriente se originó en los trabajos iniciales de la genética, realizados por Mendel, Morgan y sus seguidores. Estas investigaciones se desarrollaron en el marco de lo que se conocerá posteriormente como "genética clásica". La otra corriente fundamental de la síntesis estuvo impulsada por los trabajos en modelos matemáticos, formulados por autores como Haldane, Wright y Fisher: la genética de poblaciones (Provine, 1986). Cabe señalar que no fueron estas las únicas disciplinas involucradas en la SE, sino que participaron también otras tales como paleontología, morfología, ecología evolutiva, entre otras. Sin embargo, siguiendo aquí a autores como Arthur (1997), cabe señalar que no todas las disciplinas tuvieron los mismos roles. Mientras disciplinas tales como las mencionadas elaboraban conjuntos teóricos que buscaron dar cuenta de la diversidad biológica, otras solo debían "mostrarla". Cabe, en este sentido, citar un esquema (Figura 1) que apareció en el conocido libro de Ruse (1979) La filosofía de la biología.

\footnotetext{
3 Aunque es habitual el uso de la expresión "teoría sintética de evolución", preferimos la alternativa "síntesis evolutiva" debido a que, en rigor, no se trata de una única teoría sino más bien de un conjunto de teorías o modelos.
} 


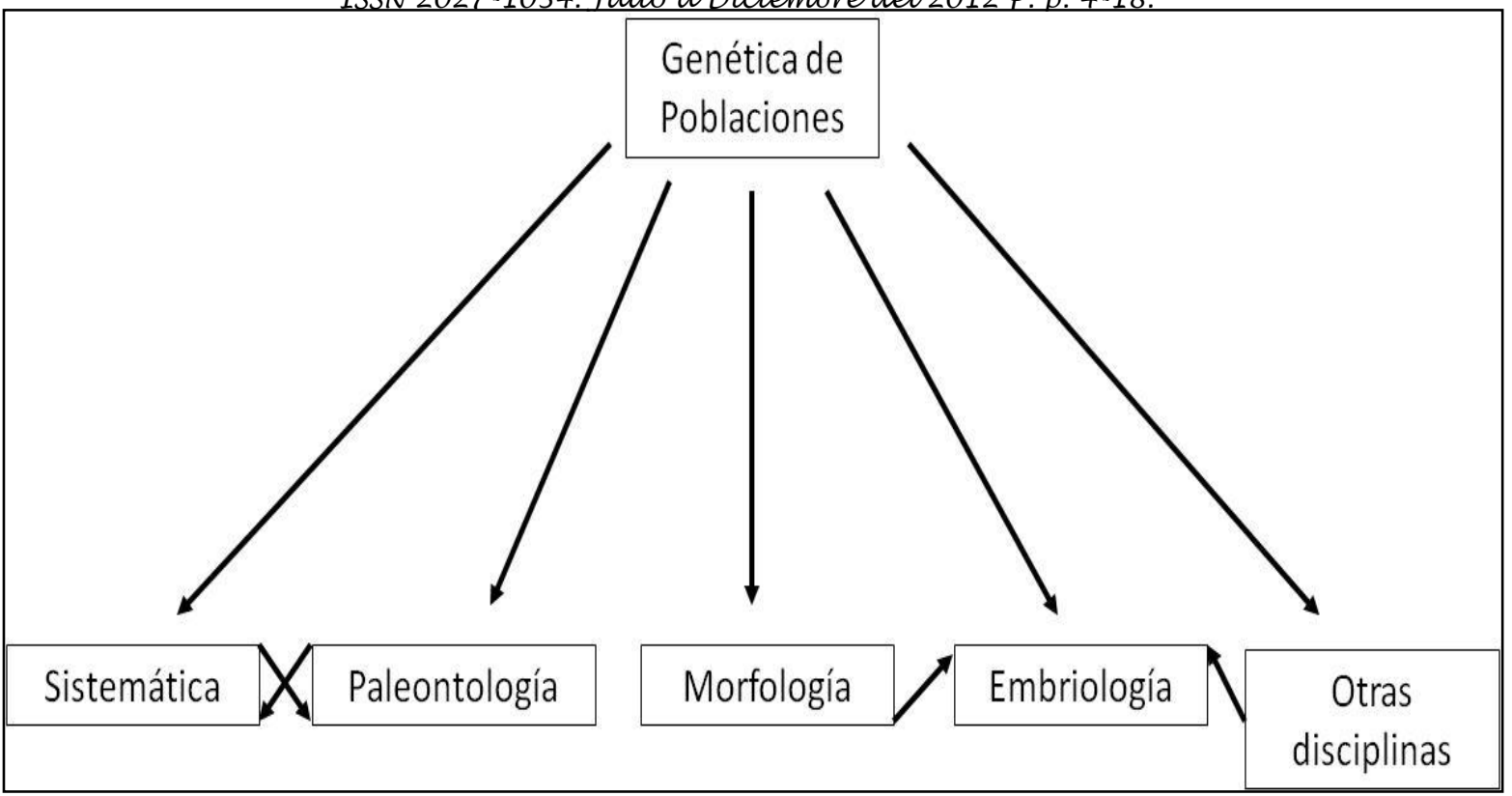

Figura 1. Figura extraída y adaptada del libro de Michael Ruse (1973) La filosofía de la biología. En esta son presentadas algunas de las disciplinas que formaron parte de la síntesis evolutiva. A su vez, son incorporadas mediante las flechas los nexos disciplinares según el autor. Cabe reconocer la relación unidireccional dada entre la genética de poblaciones y el resto.

La Figura 1 no solo representa algunas de las disciplinas involucradas, también sugiere los roles que ellas debían tener en las síntesis. Estos roles son representados por las flechas unidireccionales, que representan los cuerpos de conocimiento generados principalmente por la genética de poblaciones y que lograban explicar los cuerpos fenoménicos del resto. ¿Cuáles fueron esos conjuntos teóricos involucrados en el caso de la genética de poblaciones? Fueron teorías principalmente dirigidas a los mecanismos capaces de generar cambios evolutivos. Evidentemente, el mecanismo evolutivo más reconocido tanto dentro de los círculos académicos como educativos fue la selección natural, pero además fueron sugeridos otros tales como la migración, la deriva génica y la mutación (en el caso de que sea recurrente y con un "desvío" determinado) (Ayala, 1982). Estos mecanismos actúan de manera individual o combinada, alterando las frecuencias alélicas de una población (unidad de evolución). A su vez, cabe recordar el modo en el cual fueron recibidos dichos mecanismos: "Este paradigma newtoniano es un hogar acogedor para modelar las fuerzas evolutivas provistas por la genética de poblaciones" (Sober y Lewontin, 1982). De este modo, fue dada una conceptualización de los mecanismos como "fuerzas", con clara analogía entre la biología evolutiva y la física moderna. Estos mecanismos, según la SE, no solo lograban dar cuenta de los cambios en la microevolución (evolución de las poblaciones), sino también en la evolución de los niveles superiores al específico (macroevolución).

\section{Cambios y extensiones de la teoría evolutiva en las últimas décadas}

\section{Primera "ola" de cambios y extensiones: 1970-1990}

Esta etapa que hemos denominado como "primera ola" de cuestionamientos, ha involucrado a numerosos investigadores. Algunos de los de mayor renombre han sido Stephen Jay Gould, Richard Lewontin, Niles Eldredge, lan Tattersall, Moto Kimura y Lynn Margulis, entre otros. Sin dudas, esta corriente de investigadores fue una importante 
cuestionadora de algunos de los pilares teóricos de la síntesis evolutiva. Las críticas que han desarrollado han sido de una gran variedad y profundidad, incluyendo aspectos como el rol disciplinar de la paleontología, la necesidad de involucrar el desarrollo en los estudios evolutivos, la incorporación de la simbiosis evolutiva en la explicación de la historia de la vida y la crítica a los denominados escenarios panseleccionistas, por solo nombrar algunos de ellos. Debido a la diversidad de aspectos involucrados, que hace imposible su revisión exhaustiva en este trabajo, nos centraremos en el análisis de uno los aspectos revisados en este período que ha tenido mayor impacto en ámbitos tanto académicos como educativos: el rol de la paleontología en la teoría evolutiva y la relación entre la microevolución y la macroevolución.

Ahora bien, ¿cuáles fueron estas críticas que surgieron a partir de los finales de la década de 1960? Una de las principales fue dada a partir de si efectivamente las teorías de la genética de poblaciones podían dar cuenta de los patrones macroevolutivos. De este modo, un conjunto de autores, entre los que se desatacaba el paleontólogo Gould, asumió una relación compleja entre la microevolución y la macroevolución. Es justamente en este contexto en el que se debe entender el debate entre gradualismo y equilibrios puntuados: ¿cómo explicar las discontinuidades observadas en el registro fósil a través de la consideración solo de los mecanismos microevolutivos señalados? En la medida en que se considere, tal como señalaban estos investigadores, que las teorías microevolutivas no logran dar cuenta del registro fósil, la pregunta es: ¿cómo podremos entonces dar cuenta del ámbito de la macroevolución? Los autores de la "primera ola" sugirieron encontrar mecanismos evolutivos propios del ámbito macroevolutivo. Así, según estos autores se presenta una estructura jerárquica que postula "individuos" en diferentes niveles de la denominada jerarquía genealógica. A su vez, estos individuos tenían algunas características "semejantes" a la de los organismos biológicos. Del mismo modo, se sostuvo un tipo de mecanismo selectivo dado por la reproducción diferencial de entidades, sean genes, células, organismos, grupos de organismos o especies (Vrba y Gould, 1986; Lieberman y Vrba, 1995).

\section{Segunda "ola" de cambios y extensiones: 1990-hoy}

\section{Microevolución-macroevolución}

La propuesta de los autores de la "primera ola", presentada en la sección anterior, fue aceptada en algunos ámbitos y rechazada en otros durante las décadas siguientes. En el marco de lo que hemos entendido como la "segunda ola" de cambios y extensiones de la síntesis, en general fue aceptada la propuesta teórica generada por los investigadores de la "primera ola" en relación con el reconocimiento los límites que tenían los mecanismos microevolutivos para dar cuenta de toda la evolución biológica. Así, una parte de la comunidad científica acepta en la actualidad la propuesta de una selección en diferentes niveles, así como la incorporación de los patrones discontinuos del registro fósil, junto al conjunto teórico de la "selección multi-nivel" (Wilson, 2010). Sin embargo, esta segunda ola incluyó otros aspectos teóricos a ser revisados de la síntesis evolutiva. A continuación desarrollaremos brevemente dos de ellos: la complejización de la relación genotipofenotipo y la ampliación de las unidades de herencia y el rol del ambiente en el proceso evolutivo. 


\section{Relación entre el genotipo y el fenotipo}

Entre los elementos revisados en los últimos años, en el contexto de extensión de la SE, aparece el tipo de relación establecida entre el genotipo y el fenotipo. Ciertamente, esta crítica encuentra numerosos antecedentes, en la medida en que diversos autores venían alertando acerca de la importancia de incorporar en los estudios las interacciones entre los genes, así como entre los genes y su ambiente, sugiriendo la necesidad de reconocer la complejidad que media el genotipo y el fenotipo (ver por ejemplo Culp, 1997). En los últimos años fueron realizados estudios de diversas características al respecto, tales como los análisis acerca del grado de expresión de los genes (lo que se entiende como su "encendido") durante las sucesivas etapas del desarrollo del organismo. Este tipo de estudios ha producido un apoyo empírico a algunas consideraciones previas acerca de un desacople (al menos parcial) entre el genotipo y el fenotipo, negándose una relación lineal entre ambos (Weber, 2011). De todos modos, independientemente de si el tipo de relación establecida entre el genotipo y el fenotipo presenta una mayor o menor linealidad, sí está claro que la relación no puede ser supuesta a priori, debiéndose verificar en cada caso en particular. A su vez, estos estudios han señalado que a partir de dicho desacople "el punto de inicio" de los estudios debe ser ahora el fenotipo y no ya el gen, como ocurría claramente en el marco de la SE (Jablonka 2006). Así, por ejemplo, para una investigación biológica determinada, el supuesto correlato genético de un carácter de un grupo de organismos no puede asumirse sino que debe verificarse así como el tipo de relación que posea con el mismo.

Entre los cambios que este esquema ha implicado, aparece ni más ni menos el relativo al "hecho evolutivo". Para ello, recordemos que la noción de hecho evolutivo en la SE estaba dado por el cambio de frecuencias alélicas de una determinada población (ver por ejemplo Caponi, 2008). En cambio, desde la nueva perspectiva el hecho evolutivo estaría dado más por un cambio en el aspecto fenotípico de los seres vivos. Así, por ejemplo, un cambio evolutivo podría estar dado por un cambio en la coloración de los organismos en tanto sea heredable y afecte a más de un organismo. Desde la perspectiva de la extensión actual de la SE, deben ser incorporados necesariamente los aspectos fenotípicos en la evolución orgánica. Con el fin de responder algunas críticas acerca de que este tipo de aproximación tendría "elementos lamarckianos", es interesante reconocer cómo algunos investigadores han señalado que este tipo de aproximación, en tanto pone énfasis más en el fenotipo que en el genotipo, respondería al tipo de investigación desarrollada por el propio Darwin (ver por ejemplo West Eberhart, 2007).

\section{Ampliación de los sistemas de herencia y el rol ampliado del ambiente}

Otra de las nociones de la SE que ha sufrido importantes cuestionamientos es la relativa a los sistemas de herencia. Recordemos que para el caso de la síntesis, la unidad de herencia fundamental era el gen. En los últimos años, este escenario sufrió importantes modificaciones a partir de la propuesta de epigénesis. Ciertamente, este término fue usado con sentidos diversos, aunque en los últimos años reunió aquellos casos de herencia en los que no está involucrado el ADN (Jablonka y Lamb, 2007). La importancia de este término es tanto que no solo es indagado como concepto sino que se trata incluso de un programa de investigación centrado en el estudio de los efectos del ambiente sobre el desarrollo de los organismos, generando modificaciones fenotípicas que pueden ser heredables a través de las generaciones (Müller, 2007). Como puede suponerse, la sugerencia de que ciertos cambios fenotípicos logran transmitirse de una generación a 
otra se trata de una modificación significativa de la propuesta de la SE. En particular, los cambios epigenéticos hoy cuentan con cierto consenso en la comunidad académica, aunque no se trata de las únicas unidades de herencia involucradas. En principio, siguiendo a Jablonka y Lamb (2010), debieran considerarse tanto los sistemas de herencia genéticos y los epigenéticos, como los comportamentales. Así, el análisis de los nuevos sistemas de herencia conlleva a una reconceptualización general, quitándole la "exclusividad" al gen, no solo como la única unidad de información, sino también respecto a su rol central de ser considerado el "responsable" de las semejanzas y diferencias a través de las generaciones (ver Figura 2).

Ahora bien, el nuevo interrogante que cabe preguntarse es: ¿cuán excepcionales son estos mecanismos de herencia alternativos a los genéticos? En principio, según Jablonka y Lamb (2007), la herencia epigenética no puede ser considerada como un fenómeno infrecuente. Sin embargo, pese a las diversas evidencias empíricas, su aceptación es aún terreno de debates y disensos. Al respecto, pueden mencionarse algunos cuestionamientos desarrollados por ejemplo por Griesemer (1998) o Fox Keller (1998), tanto en lo referido a los casos analizados como ejemplos, así como también a críticas de orden teórico. Esta multiplicidad de los sistemas de herencia, así como su estructuración jerárquica, son algunas de las modificaciones principales que se han dado en los pilares de la SE.

\section{Sistemas de herencia Epigenético Genético Comportamental Lingüístico

Medio de transmisión $\begin{gathered}\text { Cromatina, } \\ \text { circuitos }\end{gathered}$ ADN $\begin{gathered}\text { Aprendizaje } \\ \text { social, etc. }\end{gathered}$ metab, etc.}

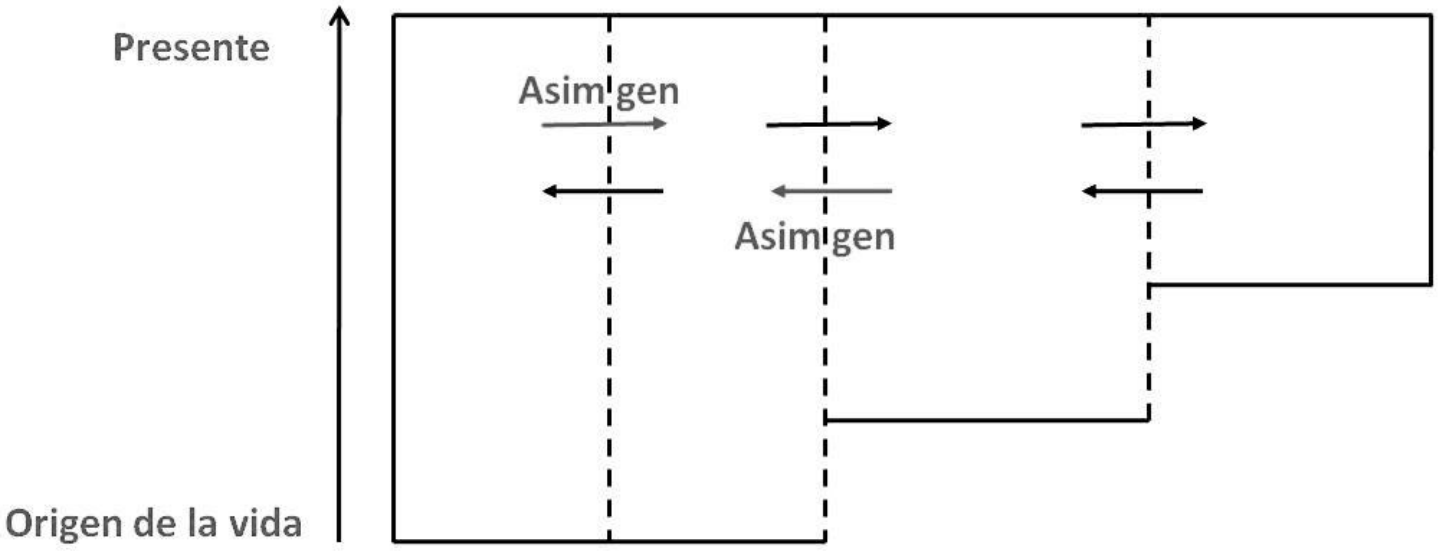

Figura 2. Gráfico extraído y adaptado de Jablonka, Lamb y Avital (1998). Se representan diferentes sistemas de herencia y sus interacciones. Asim genética: asimilación genética.

En los últimos años, respecto a la propuesta de la SE, también fue revisado el rol del ambiente. Recordemos que en el caso de la SE, el ambiente "actuaba" como una especie de "filtro" de la variabilidad que se originaba, por ejemplo, de la mutación genética. Así, aquellos "monstruos" que no tenían esperanza en sobrevivir "quedaban en el camino", mientras que otros que presentaban características favorables novedosas en relación con el ambiente que les tocaba vivir, podrían dejar una mayor cantidad de descendientes. A 
partir de la extensión de la SE, el ambiente no solo es conceptualizado como un "filtro" de la diversidad de lo viviente, sino también como capaz de generar estímulos que puedan alterar aspectos del organismo durante su desarrollo, "dirigiendo" su cambio. Este nuevo "rol" del ambiente, que lo involucra también en el origen de la variación heredable, ha sido denominado "inducción ambiental". En algunos casos, estos cambios fenotípicos podrían ofrecer algún tipo de beneficio a sus portadores dando lugar a un escenario adaptativo. Ciertamente, la "inducción ambiental" presentó algunas "ventajas" epistémicas respecto a las propuestas anteriores. Una de las principales es su capacidad de explicar procesos rápidos, en la medida en que son afectados una gran cantidad de individuos en una población determinada, sin tener que "esperar" el origen de determinada variante a partir de un proceso aleatorio generador de variabilidad (Jablonka, 2006). De este modo, a partir del "extendido" rol del ambiente, se reconoce cómo, pese a la presencia de una diversidad de genotipos en una determinada población en un determinado ambiente, pueden generarse fenotipos similares (Scott, 2002). Tal como hemos visto, la propuesta de los procesos evolutivos presentes en algunas de las versiones extendidas de la SE, han alterado pilares que caracterizaron a los procesos evolutivos en décadas pasadas. Sus consecuencias, tal como hemos visto, son múltiples y generan grandes alteraciones conceptuales en diversos campos del estudio de lo viviente.

\section{Implicancias didácticas}

\section{Consideraciones y argumentos acerca de la inclusión (o no) de los debates actuales acerca de la extensión de la teoría evolutiva en la enseñanza de la biología}

Como se mencionó al comienzo de este artículo, la "síntesis" supuso la integración de la teoría de la evolución de Darwin con el resto de las disciplinas biológicas, especialmente la genética. Una de las piedras angulares del consenso que constituyó la SE fue el reconocimiento del rol central que la selección natural juega en la evolución biológica (Bowler, 2003). Algunos de los nuevos desarrollos teóricos reseñados en las secciones anteriores cuestionan en cierta medida este lugar central o exclusivo de la selección natural. En relación con este punto, consideramos necesario aclarar que no hay dudas acerca de la necesidad de enseñar los principios de la SE, especialmente el modelo de evolución por selección natural (MESN). Esta certeza se debe a que en cualquier versión concebible actualmente de la teoría evolutiva (aun aquellas que suponen grandes revisiones) la selección natural sigue ocupando un rol central (Dupré, 2006), y a que el MESN constituye la única explicación aceptada actualmente de la adaptación biológica (Futuyma, 2009; Ridley, 2003; Sterelny y Griffiths, 1999). La pregunta es entonces qué otros modelos habría que enseñar además del MESN.

En este sentido, algunos autores han criticado la tendencia en la enseñanza general a identificar "evolución" con "selección natural". Catley (2006), por ejemplo, señala que en la enseñanza general solo se tratan los procesos microevolutivos, en detrimento de los macroevolutivos y de los principales eventos de la historia de la vida. Esta crítica es atendible aún en caso de no abandonar la SE como referencia, ya que los contenidos que según Catley son desatendidos forman parte de la SE. Así, además de evaluar la necesidad de enseñar los nuevos modelos, cabe preguntarse qué conceptos de la propia SE han sido excluidos de la enseñanza general.

La pregunta que surge en relación con los nuevos modelos reseñados en las secciones anteriores y con los debates asociados es, entonces, en qué medida estaría justificada su 
inclusión en la enseñanza. Claramente, en toda respuesta sobre qué enseñar se harán visibles también las diferentes finalidades involucradas en la enseñanza de las ciencias (Meinardi, 2010).

\section{Argumentaciones I: la estructura de la biología evolutiva}

Un posible criterio para la selección de contenidos, centrado en la lógica disciplinar, supone privilegiar la enseñanza de aquellos modelos más generales, que abarcan muchos submodelos y conceptos (Jiménez Aleixandre y Sanmartí, 1997). Es difícil describir la "teoría de la evolución" como una teoría unificada que pueda reducirse a una serie de enunciados lógicamente relacionados, como se espera desde una visión sintáctica de las teorías científicas. Algunos autores (Lloyd, 1994; Thompson, 1989) proponen que esta teoría es mejor caracterizada como un amplio conjunto de modelos, como se sugiere desde una perspectiva semántica. Así, un primer paso para decidir qué modelos enseñar sería elucidar la "trama conceptual" de la disciplina de referencia (Astolfi, 2001). Este marco teórico permitiría evaluar la importancia relativa de los distintos modelos de acuerdo, por ejemplo, con el número de conexiones que cada modelo establece con los demás. Podemos preguntarnos: ¿se ha hecho tal análisis para el caso de la biología evolutiva?

Desde esta perspectiva, centrada en la lógica disciplinar, cabe preguntarse por el estatus de los nuevos modelos. Es decir, en qué medida están consolidados, en qué medida existe consenso mínimo en la comunidad de expertos sobre su validez. Giere (1992) sugiere que para saber cuáles son los modelos relevantes de una disciplina científica hay que remitirse a los libros de texto universitarios, porque es a partir de estos textos que los estudiantes aprenden en qué consiste su disciplina. Sin embargo, podría objetarse que este consejo nos limita a tomar como referencia disciplinar la versión más ortodoxa de una teoría. Es esperable, por la propia dinámica de la construcción del conocimiento científico, que los nuevos modelos sean cuestionados por buena parte de la comunidad de expertos y que en el futuro algunos de estos modelos se consoliden mientras que otros serán olvidados. Sin embargo, este juicio solo está disponible a posteriori. Estas consideraciones plantean algunos interrogantes: ¿la decisión de enseñar o no un modelo sobre el cual no existe consenso en la comunidad de expertos es parte de las atribuciones de un docente en tanto que profesional autónomo? Dado el limitado tiempo disponible para enseñar los modelos bien establecidos, ¿es deseable dedicar tiempo a enseñar modelos que en pocos años podrían revelarse inadecuados? En principio, teniendo en cuenta estas consideraciones, parecería poco justificado dedicar tiempo en la escuela a enseñar modelos que no gozan de un mínimo consenso en la comunidad de expertos. Esta conclusión se refuerza si consideramos que los resultados en términos de aprendizaje de aquellos modelos sobre cuya importancia existe un acuerdo total, son claramente deficientes. En general, el tiempo dedicado a la enseñanza de un contenido se correlaciona con los logros de aprendizaje, por lo que parecería más razonable dedicar todo el tiempo posible a la enseñanza de los modelos básicos de la biología evolutiva.

Los modelos científicos son construcciones elaboradas con el objetivo de dar cuenta de ciertos fenómenos. Desde la perspectiva de la alfabetización científica, se espera que el ciudadano "científicamente alfabetizado" pueda dar cuenta del mundo a partir de modelos teóricos (Acevedo Díaz, 2004). Así, cabe preguntarse también de qué fenómenos dan cuenta los nuevos modelos. Para decirlo de otro modo, ¿qué fenómenos podrían comprender los estudiantes gracias al aprendizaje de estos modelos? ¿Son relevantes 
estos fenómenos para la población general? Este análisis epistemológico de los nuevos modelos también arrojaría luz sobre su relevancia para la enseñanza general.

\section{Argumentaciones II: prácticas sociales y naturaleza de la ciencia}

La lógica interna de una disciplina de referencia no constituye el único criterio posible para seleccionar los contenidos a enseñar, más aún en aquellos casos que existen importantes disensos en el seno de la comunidad académica. También podrían considerarse las "prácticas sociales de referencia". De acuerdo con Astolfi (2001), se trata de examinar de qué manera pueden servir de referencia a las actividades científicas escolares las de producción, las de ingeniería y las domésticas, entre otras. Por ejemplo, desde una perspectiva centrada en la estructura lógica de la disciplina, podría ser poco relevante introducir en la enseñanza obligatoria la discusión sobre el concepto de raza. Sin embargo, su inclusión estaría justificada dadas las fuertes implicancias sociales de este concepto. Nos preguntamos entonces, ¿cuál es la relevancia de los nuevos modelos más allá de la lógica disciplinar? Por ejemplo, considerando que las nuevas propuestas cuestionan la linealidad de la relación entre genotipo y fenotipo, ¿serviría la inclusión de estos contenidos para favorecer una mirada crítica sobre lo que Fox Keller (2002) ha denominado "el discurso del gen" y, en general, sobre las diversas formas del determinismo genético?

A su vez, podría argumentarse que el problema no solo es qué modelos se enseñan, sino también cómo se enseñan. Es decir, no sería cuestionable enseñar un modelo en discusión siempre y cuando los estudiantes comprendan claramente cuál es el estatus del modelo en cuestión. Desde la perspectiva de la naturaleza de la ciencia, de hecho, podría considerarse conveniente tratar estos modelos, ya que esto permitiría que los estudiantes construyeran una visión de la ciencia que incluye el disenso y el debate como aspectos centrales del quehacer científico. Tanto estudiantes como profesores suelen participar de una visión distorsionada de la naturaleza de la ciencia (Adúriz Bravo, 2005). Esta visión supone que el conocimiento científico consiste en un conjunto de enunciados verdaderos sobre el mundo, firmemente establecidos sobre evidencias experimentales (Sanmartí, 2002). En esta visión no tienen lugar el disenso, el debate, la ambigüedad o incerteza. De hecho, esta visión distorsionada de la ciencia es explotada por algunos movimientos religiosos (creacionismo) que muestran la existencia de debates en el seno de la biología evolucionista como una presunta debilidad. Del mismo modo, sostienen que la teoría de la evolución es "solo una teoría", sugiriendo -falazmente- que es el mismo hecho de la evolución el que está en discusión (NAS, 2008). Desde esta perspectiva insisten en la necesidad de "enseñar la controversia" (Scott y Branch, 2003). Aunque claramente falaz, ya que las controversias giran en torno a cómo y por qué se produce la evolución y no en torno a si se produce o no (NAS 2004), esta estrategia retórica es eficaz porque cuenta con una errónea visión de la ciencia de la que participa el público. Por estos motivos, mostrar a los estudiantes que en el interior de la biología evolutiva tienen lugar debates, contribuiría a la construcción de una imagen de ciencia más adecuada. En el caso de la biología evolutiva los estudiantes deberían comprender que tienen lugar numerosos debates y que esto es un signo de la vitalidad de esta disciplina, al tiempo que comprenden que estos debates no suponen poner en duda el hecho de la evolución (NAS, 2004). Desde la perspectiva de la naturaleza de las ciencias, entonces, podría suponerse que la inclusión de estos debates sería positiva e, incluso, necesaria. 


\section{Otras consideraciones didácticas}

A su vez, hay al menos otros dos aspectos que sin duda cumplen funciones importantes en este contrapunto: la transposición didáctica y los niveles educativos involucrados.

\section{La transposición didáctica}

En un famoso artículo, Francis Halbwachs (1975) mostró que la física del físico es diferente de la del profesor y de la del alumno. Esto no es algo negativo, ya que estas distintas instancias suponen diferentes fines e intereses. El hecho de que estos saberes sean diferentes evidencia que un objeto de saber sufre una serie de transformaciones para convertirse en un objeto de enseñanza, proceso denominado "transposición didáctica" (Chevallard, 2005). Por ello se puede hablar de una "ciencia escolar", diferente de la "ciencia erudita" (Sanmartí, 2002).

Como sucede para la mayoría de las disciplinas escolares, la biología evolutiva que se enseña en las escuelas es producto de un proceso de transposición que tuvo lugar hace muchos años. En este caso en particular, el referente teórico es la SE, una teoría consolidada a mediados del siglo XX. Esto supone que algunos de los aspectos de esta "teoría de la evolución escolar" podrían ser cuestionados tanto desde una perspectiva biológica como didáctica.

Según Chevallard (2005), la transposición didáctica consta de dos etapas. En la primera, la "noosfera" (científicos interesados por la enseñanza, autores de libros de texto, didactas) convierte el "saber sabio" en un "saber a enseñar". En la segunda etapa, este es convertido en un "saber enseñado" por el profesorado. Podríamos preguntarnos qué tanto de este trabajo ha sido realizado en el caso de los nuevos modelos de la biología evolutiva. Es evidente que los modelos descritos en la sección "Extensión de la síntesis" no han sido aún convertidos en un "saber a enseñar". Es decir, no disponemos de transposiciones didácticas de los nuevos modelos de la biología evolutiva. Si esto es cierto para el nivel universitario, es más cierto aún para el nivel secundario. Al menos en Argentina, los únicos modelos que pueden encontrarse en algunos libros de texto para enseñanza secundaria y que, en algún grado y sentido, van más allá del énfasis en la selección de la SE, son los aportes de Stephen Jay Gould (fundamentalmente el modelo de equilibrios puntuados) y de Lynn Margulis (endosimbiosis). Así, en caso de que consideráramos que los nuevos modelos merecen ser enseñados, deberíamos enfrentar el problema de la ausencia de un adecuado proceso de transposición didáctica, es decir, la falta de versiones de estos nuevos modelos accesibles para el profesorado. Esta situación vale para otras disciplinas escolares (Sanmartí, 2002) y es parcialmente responsable del "envejecimiento" del saber escolar (Chevallard, 2005), es decir, del hecho de que los contenidos escolares resulten "desactualizados" a los ojos de los expertos y de la comunidad en general. Desde esta perspectiva, podría suceder que la comunidad educativa no estuviera aún en condiciones de incorporar los nuevos modelos a la enseñanza general.

\section{Niveles educativos involucrados}

La mayoría de las reflexiones previas estuvieron centradas en la necesidad, conveniencia o factibilidad de enseñar los nuevos conceptos de la biología evolutiva en la enseñanza secundaria obligatoria. Es decir, la población de estudiantes que tenemos en mente está 
constituida mayoritariamente por personas que no seguirán estudios superiores relacionados con la biología (y menos aún con la biología evolutiva). Es esperable que las posibles respuestas a las preguntas planteadas cambien cuando se tienen en cuenta poblaciones de estudiantes más particulares, concretamente futuros biólogos y futuros profesores de biología.

Aunque no hemos hecho estudios sobre el tema y, hasta donde sabemos, no existen tales estudios, sugerimos, basándonos en nuestras experiencias y conocimientos personales, que las nuevas temáticas de biología evolutiva reseñadas en este artículo son en gran medida ignoradas en la formación docente e, incluso, en la formación de los futuros biólogos ¿La discusión de estos modelos debería ser parte de la formación de los futuros biólogos? Aún en este ámbito educativo, tal vez no sea defendible la necesidad y factibilidad de enseñar todos los modelos existentes. Muchos son tan específicos que solo deberían ser parte de cursos superiores dirigidos a investigadores en el área en cuestión. Por ejemplo, el "modelo de umbral de poliginia" (Krebs y Davis, 1993), que versa sobre la evolución de los sistemas de apareamiento, no sería pertinente para un investigador especializado en biología molecular. Sin embargo, los nuevos modelos reseñados en este artículo afectan algunos de los supuestos principales de la SE. Dado que la SE ocupa un lugar central en las ciencias biológicas, consideramos que los futuros biólogos deberían tener un mínimo acercamiento a estos modelos que les permitiera dar cuenta de los cambios que está sufriendo la biología evolutiva.

Esta reflexión vale también para la formación de los futuros profesores de biología. Además, en este caso, los estudiantes se beneficiarían de la necesaria reflexión sobre la naturaleza de la ciencia (Adúriz Bravo, 2005) por las mismas razones ya expuestas para los estudiantes de nivel secundario. Este punto es particularmente relevante en el caso de los futuros profesores, ya que la visión distorsionada de la naturaleza de las ciencias por parte del profesorado constituye uno de los factores que condicionan negativamente la enseñanza de las ciencias y que contribuyen al desinterés de los estudiantes en relación con estas disciplinas.

\section{Apuntes finales}

En el recorrido realizado hemos podido reconocer algunas de las modificaciones y extensiones teóricas que ha tenido la síntesis evolutiva en los últimos años. En particular, nos hemos centrado en aquellas que se han dirigido a las dificultades que tienen las teorías que dan cuenta de la variación y evolución genotípica para dar cuenta de la evolución fenotípica. A su vez, se han presentado argumentaciones diversas que conducen o no a la incorporación de dichas modificaciones en la enseñanza de la teoría evolutiva, principalmente en la escuela media. Sin duda, independientemente de la decisión final que se considere al respecto, estos debates abiertos presentan una oportunidad para reconocer aspectos de interés en relación con la naturaleza de las ciencias, así como para reflexionar sobre las diversas dificultades asociadas a la eventual inclusión de estos nuevos contenidos en la enseñanza. Ciertamente, no ha sido nuestro objetivo generar conclusiones definitivas al respecto (tampoco podríamos hacerlo), sino más bien aportar algunos elementos que, creemos, contribuyen a un debate significativo y necesario. 


\section{Referencias}

Acevedo Días, J. (2004). Reflexiones sobre las finalidades de la enseñanza de las ciencias: educación científica para la ciudadanía. Revista Eureka sobre Enseñanza y Divulgación de las Ciencias, 1, 3-16.

Adúriz Bravo, A. (2005). La naturaleza de la ciencia. En Merino Rubilar, C., Gómez Galindo, A. y Adúriz Bravo, A. Áreas y estrategias de investigación en la didáctica de las ciencias experimentales. Vol. I. Barcelona: Universidad Autónoma de Barcelona.

Alters, B. y Nelson, C. (2002). Perspective: teaching evolution in higher education. Evolution, 56, 1891-1901.

Arthur, W. (1997). The Origin of Animal Body Plans: A Study in Evolutionary Developmental Biology [El origen de los planes corporales de los animales: un estudio en biología del desarrollo].Cambridge MA: Cambridge University Press.

Ayala F. J. (1982). Beyond Darwinism? The challenge of macroevolution to the synthetic theory of evolution. Philosophy of Science, 2, 275-291.

Bowler, P. (2003). Evolution. The History of an Idea [Evolución. La historia de una idea] ( $3^{\mathrm{a} E d}$.). Berkeley: University of California Press.

Catley, K. (2006). Darwin's Missing Link-A Novel Paradigm for Evolution Education. Science Education, 5, 767-783.

Caponi, G. (2008). El segundo pilar. La biología evolucioniaria desenvolvimiental y el surgimiento de una teoría complementaria a la teoría de la selección natural. Ludus Vitalis, 29, 3-32.

Chevallard, Y. (2005). La transposición didáctica. Del saber sabio al saber enseñado. Buenos Aires: Aique.

Culp, S. (1997).Establishing Genotype/Phenotype Relationships: Gene Targeting as an Experimental Approach, Philosophy of Science, 64, 268-278.

Dupré, J. (2006). El legado de Darwin. Qué significa hoy la evolución. Buenos Aires: Katz.

Fox Keller, E. (2002). El siglo del gen. Cien años de pensamiento genético. Barcelona: Península.

Fox Keller, E. (1998). Structures of Heredity. Biology and Philosophy, 13, 113-118.

Futuyma, D. (2009). Evolution [Evolución]. Sunderland: Sinauer.

Giere, R. (1992). La explicación de la ciencia. México: Consejo Nacional de Ciencia y Tecnología. 
Griesemer, J. (1998). Turning Back to Go Forward. A review of Epigenetic Inheritance and Evolution. The Lamarckian Dimension, by Eva Jablonka and Marion Lamb. Biology and Philosophy, 13, 103-112.

Gould, S. (2004). La estructura de la teoría evolutiva. Barcelona: Tusquets.

Halbwachs, F. (1975). La physique du maitre entre la physique du physicien et la physique de l'eleve. Reveu française de pédagogie, 33, 19-29.

Jablonka E. (2006). Genes as followers in evolution - a post-synthesis synthesis? Biology and Philosophy, 21, 143-154.

Jablonka, E. y Lamb, M. (2007). The expanded evolutionary synthesis - a response to Godfrey-Smith, Haig, and West-Eberhard. Biology and Philosophy, 22, 453-472.

Jablonka, E. y Lamb, M.J. (2010). Transgenerational Epignetic Inheritance. En M. Pigliucci y G. Müller (Eds.), Evolution. The extended Synthesis (pp. 137-174). Cambridge: MIT Press.

Jablonka, E., Lamb, M. y Avital, E. (1998). Lamarckian Mechanisms in Darwinian Evolution. Trends in Ecology \& Evolution, 13, 206-210.

Jiménez Aleixandre, M. y Sanmartí, N. (1997). ¿Qué ciencia enseñar? Objetivos y contenidos en la educación secundaria. En L. del Carmen. La enseñanza y el aprendizaje de las ciencias de la naturaleza en la educación secundaria. Barcelona: ICE / Horsori.

Krebs, J. y Davis, N. (1993). Behavioral Ecology [Ecología comportamental]. Oxford: Blackwell Science.

Lieberman, B.S. y Vrba, E.S. (1995). Hierarchy Theory, Selection, and Sorting. Bioscience, 45, 394-399.

Lloyd, E. (1994). The Structure and Confirmation of Evolutionary Theory [Estructura y confirmación de la teoría evolucioniaria]. Princeton: Princeton University Press.

Mayr, E. (1992). Una larga controversia: Darwin y el darwinismo. Barcelona: Crítica.

Meinardi, E. (2010). El sentido de educar en ciencias. En E. Meinardi. Educar en ciencias (pp. 15-38). Buenos Aires: Paidós.

Müller, G.B. (2007), Evo-devo: Extending the Evolutionary Synthesis. Nature Reviews Genetics, 8, 943-949.

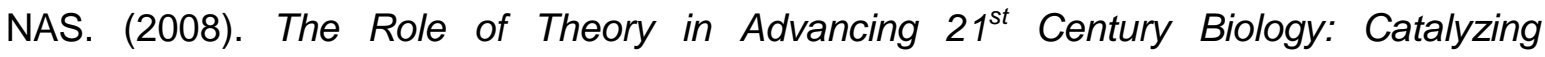
Transformative Research [El rol de la teoría en la biología del siglo 21: catalizando investigación transformativa]. Washington, DC: National Academy Press.

NAS. (2004). Teaching about Evolution and the Nature of Science [Enseñar sobre la evolución y la naturaleza de las ciencias]. Washington, DC: National Academy Press. 
Provine, W.B. (1986). Sewall Wright and Evolutionary Biology. Chicago: University of Chicago Press.

Ridley, M. (2003). Evolution [Evolución]. Third edition. New York: Wiley-Blackwell.

Ruse, M. (1994). Tomándose a Darwin en serio. Barcelona: Salvat.

Ruse, M. (1979). La filosofía de la biología. Madrid: Alianza Editorial.

Sanmartí, N. (2002). Didáctica de las ciencias en la educación secundaria obligatoria. Madrid: Síntesis.

Scott, E. y Branch, G. (2003). What's wrong with "teaching the controversy. TRENDS in Ecology and Evolution, 18, 499-502.

Scott, R.J. (2002). How developmental is evolutionary developmental biology? Biology and Philosophy, 17,591-611.

Smith, M. (2010a). Current Status of Research in teaching and Learning Evolution: I. Philosophical/Epistemological Issues. Science and Education, 6-8, 523-538.

Smith, M. (2010b). Current Status of Research in teaching and Learning Evolution: II. Pedagogical Issues. Science and Education, 6-8, 539-571.

Sober, E.R. y Lewontin. R.C. (1982). Artifact, Cause and Genetic Selection. Philosophy of Science, 49, 157-180.

Stamos, D. (2009). Evolución. Los grandes temas: sexo, raza, feminismo, religión y otras cuestiones. Barcelona: Biblioteca Buridán.

Sterelny, K. y Griffiths, P. (1999). Sex and Death. An Introduction to Philosophy of Biology [Sexo y muerte. Una introducción a la filosofía de la biología]. Chicago: The University Chicago Press.

Thompson, P. (1989). The Structure of Biological Theories [La estructura de las teorías biológicas]. Albany: State University of New York Press.

Vrba, E.S. y Gould, S.J. (1986). The hierarchical expansions of sorting and selection: sorting and selection cannot be equated. Paleobiology, 12, 217-228.

Weber, B.H. (2011). Extending and expanding the Darwinian synthesis: the role of complex systems dynamics. Studies in History and Philosophy of Biological and Biomedical Sciences, 42, 75-81.

West-Eberhard, M.J. (2007). Dancing with DNA and flirting with the ghost of Lamarck. Biology and Philosophy, 22, 439-451.

Wilson, D.S. (2010). Multilevel selection and major transitions. En M. Pigliucci y G. Müller (Eds.), Evolution. The extended Synthesis [Evolución. La síntesis extendida] (pp. 81-94). Cambridge: MIT Press. 